\title{
CADM2 inhibits human glioma proliferation, migration and invasion
}

\author{
NAN LIU ${ }^{1 *}$, CHEN YANG $^{2,3^{*}}$, WANSHENG BAI ${ }^{4 *}$, ZHEN WANG $^{1}$, XIN WANG $^{5}$, MARK JOHNSON $^{6}$, \\ WENSHI WANG ${ }^{7}$, PENGXING ZHANG ${ }^{1}$, HONGWEI YANG ${ }^{6}$, HUI LIU ${ }^{1}$, \\ YINGDUAN CHENG ${ }^{1}$ and YANYANG TU ${ }^{1,5}$ \\ ${ }^{1}$ Department of Experimental Surgery, Tangdu Hospital, Fourth Military Medical University, Xi'an, Shaanxi 710038; \\ ${ }^{2}$ Postdoctoral Research Station of Neurosurgery, Wuhan General Hospital of Guangzhou Command, \\ The People's Liberation Army, Wuhan, Hubei 430064; ${ }^{3}$ Department of Neurosurgery, Tangdu Hospital, \\ Fourth Military Medical University, Xi'an, Shaanxi 710038; ${ }^{4}$ Department of Neurosurgery, \\ 323rd Hospital of The People's Liberation Army, Xi'an, Shaanxi 710054, P.R. China; \\ ${ }^{5}$ Department of Neurosurgery, Brigham and Women's Hospital, Harvard Medical School, Boston, \\ MA 02115; ${ }^{6}$ Department of Neurosurgery, University of Massachusetts Medical School, \\ Worcester, MA 01605; ${ }^{7}$ HemaCare, Van Nuys, CA 91406 , USA
}

Received August 25, 2017; Accepted September 12, 2018

DOI: 10.3892/or.2019.7010

\begin{abstract}
Malignant glioma is one of the most common malignant tumors in the brain parenchyma with a poor prognosis. Cell adhesion molecules (CADMs) immunoglobulin super family is involved in the maintenance of cell adhesion, polarity and tumor suppression. However, the role and mechanisms of CADM2 in human glioma have yet to be elucidated. Therefore, the present study evaluated the expression level of CADM2 and demonstrated that CADM2 was markedly downregulated in human glioma tissues compared with normal brain tissue and glioma cell lines, and the CADM2 expression level was significantly decreased in high-grade glioma tissues. Overexpression of CADM2 inhibited the proliferation of glioma cell proliferation in vitro and in vivo. CADM2 also inhibited the migration and invasion of U87 and U251 cells. Furthermore, overexpression of CADM2 induced a significant decrease in the expression of G1/S transition key regulators, cyclin D1, cyclin E, cyclin-dependent kinase (CDK)2 and CDK4. Additionally, CADM2 expression was associated with alterations in epithelial-mesenchymal transition (EMT) markers, including E-cadherin and $\beta$-catenin. Taken together,
\end{abstract}

Correspondence to: Professor Yanyang Tu or Mr. Yingduan Cheng, Department of Experimental Surgery, Tangdu Hospital, Fourth Military Medical University, Xin Si Road, Xi'an, Shaanxi 710038, P.R. China

E-mail: ayonst@163.com

E-mail: cyd116@hotmail.com

*Contributed equally

Key words: cell adhesion molecule 2, glioma, proliferation, migration, invasion the results of the present study demonstrated that CADM2 inhibits glioma tumorigenesis by regulating the cell cycle and the EMT process, suggesting that CADM2 may be a novel potential therapeutic target in human glioma.

\section{Introduction}

Glioma is one of the most common primary malignant tumors in the central nervous system (CNS), which accounted for $\sim 40 \%$ of all intracranial tumors (1). According to the world health organization (WHO), gliomas can be divided into four grades (I, II, III and IV) (2). Glioblastoma (GBM), the highest grade (grade IV) of glioma tumor, is the most malignant form of astrocytoma. Although there have been great advances in treatment options, including surgery, radiotherapy and chemotherapy, in recent decades, the prognosis of GBM remains very poor. At present, the 5-year survival rate of patients with GBM is only $9.8 \%$ (3-5). In order to improve the prognosis of patients with GBM, it is urgent to identify novel therapeutic targets.

Cell adhesion molecules (CADM) consists of a protein family, which has been reported to maintain cell polarity. The majority of the CADMs belong to the immunoglobulin superfamily, and recent research has suggested that CADM also acts as a tumor suppressor (6-9). CADM2 is a member of the CADM family, and previous studies have demonstrated that CADM2 inhibits human renal clear cell carcinoma by promoting DNA promoter methylation and/or loss of heterozygosity. Loss of CADM2 causes higher tumor pathological stages (6). In addition, CADM2 has been observed to be downregulated in prostate (10) and ovarian cancer (11), and lymphoma and melanoma $(12,13)$. However, to the best of our knowledge, the present study was the first to have reported the role of CADM2 in human glioma. In the present study, the expression level of CADM2 in human glioma was analyzed by bioinformatics (Oncomine database) and tissue microarrays. The results 
demonstrated that CADM2 was markedly downregulated in human glioma tissues. The expression level of CADM2 was decreased, while glioma pathological grades were increased. CADM2 inhibited GBM cell proliferation, migration and invasion in vitro and in vivo. Furthermore, CADM2 regulated the glioma cell G1/S transition and affected glioma cell epithelial-mesenchymal transition (EMT). Taken together, the results of the present study implicated CADM2 as a novel tumor suppressor candidate in human glioma, and provided a novel molecular target for the treatment of human glioma.

\section{Materials and methods}

Tissues. Human glioma tissues were obtained from the surgical branch of Tangdu Hospital, Fourth Military Medical University, China. Normal brain tissues, which were preserved in liquid nitrogen, were obtained from the patients who had undergone partial brain resection. The tissue samples were obtained with the written informed consent of the patients, and the process was approved by the Ethics Committee of Tangdu Hospital Institutional Review Board.

Cell lines and lentiviral transfection. The established U251 and U87MG cell lines used in the present study were purchased from the Cell Bank of Chinese Academy of Sciences (14). The authenticity of the cancer cell lines was tested by short tandem repeat (STR) profiling. The STR result demonstrated that the U87 cell line used in the present study matched the U87MG cell from ATCC. However, the U87MG cell line from ATCC may not be the original GBM cell line from the University of Uppsala established in 1968. It has been reported that the U87MG cell line from ATCC is of CNS origin and is likely to be derived from another patient with glioma, although its source is unknown (15). Therefore, the aforementioned misidentifications of U87MG-ATCC did not affect the outcomes of the present study. All the cell lines were cultured in $10 \%$ fetal bovine serum (FBS) Dulbecco's modified Eagle's medium (DMEM; both Gibco; Thermo Fisher Scientific, Inc., Waltham, MA, USA). The CADM2 coding sequence (CDS) was sub-cloned into a pCDH1-overexpression vector using the restriction sites of Bamhl and Xhol. U251 and U87 cells were transfected with CADM2-overexpression lentivirus, and stable CADM2-overexpression cell lines were established by puromycin screening at $2 \mu \mathrm{g} / \mathrm{ml}$.

Reverse transcription-quantitative polymerase chain reaction $(R T-q P C R)$. Total RNA was extracted using TRIzol reagent (Thermo Fisher Scientific, Inc.), according to the manufacturer's protocol. Reverse transcription was performed using a Takara reverse transcription kit (Takara Bio, Inc., Otsu, Japan). The sequences of the primers used were as follows: CADM2 forward, 5'-CCTCAATGCCACCCCTCAG-3' and reverse, 5'-TTCTCCGCCATCCTTTGTCC-3'; and $\beta$-actin forward, 5'-TCCCTGGAGAAGAGCTACG-3' and reverse, 5'-GTA GTTTCGTGGATGCCACA-3'. The reverse transcription process was as follows: $37^{\circ} \mathrm{C}$ for $1 \mathrm{~h}$ and $85^{\circ} \mathrm{C}$ for $15 \mathrm{sec}$. PCR conditions were $95^{\circ} \mathrm{C}$ for $5 \mathrm{~min}$, followed by 40 cycles at $95^{\circ} \mathrm{C}$ for $20 \mathrm{sec}, 60^{\circ} \mathrm{C}$ for $30 \mathrm{sec}, 72^{\circ} \mathrm{C}$ for $60 \mathrm{sec}$. Gene expression in each sample was normalized to relative to that of $\beta$-actin mRNA. qPCR was performed using SYBRGreen (Takara Bio,
Inc.) with the ABI PRISM 7700 Sequence Detection system (Applied Biosystems; Thermo Fisher Scientific, Inc.). The expression was normalized to human $\beta$-Actin expression using the $2^{-\Delta \Delta \mathrm{Cq}}$ method (16).

Western blot assay. Radioimmunoprecipitation assay (RIPA) lysis buffer was used to lyse glioma tissues and different glioma cell lines, and complete protease inhibitor cocktail (Beyotime Institute of Biotechnology, Haimen, China) was supplemented with RIPA lysis buffer prior to lysis. Bicinchoninic acid protein assay kit (Beyotime Institute of Biotechnology) was used to detect protein concentration. A total of $30 \mu \mathrm{g}$ protein samples were loaded onto the gel and separated by $12 \%$ SDS-PAGE gel. Protein was subsequently transferred to polyvinylidene difluoride membranes. Following blocking with 5\% skimmed milk for $1 \mathrm{~h}$ at room temperature, the membranes were incubated with the following primary antibodies at $4^{\circ} \mathrm{C}$ overnight: CADM2 (dilution, 1:4,000; cat. no. ab64873; Abcam, Cambridge, UK), $\beta$-actin (dilution, 1:4,000; cat. no. M20011; Abmart Co., Ltd., Shanghai, China), CDK2 [dilution, 1:2,000; cat. no. 2546; Cell Signaling Technology (CST)], CDK4 (dilution, 1:2,000; cat. no. 12790; CST), E-cadherin (dilution, 1:2,000; cat. no. 14472; CST), $\beta$-catenin (dilution, 1:2,000; cat. no. 8480; CST), cyclin D1 (dilution, 1:1,000; cat. no. sc-70899; Santa Cruz Biotechnology, Inc., Dallas, TX, USA) and cyclin E (dilution, 1:1,000; cat. no. sc-377100; Santa Cruz Biotechnology, Inc.). On the second day, the membranes were incubated with horseradish peroxidase (HRP)-conjugated secondary antibodies (goat anti-mouse IgG, cat. no. ab97023 or goat anti-rabbit IgG, cat. no. ab6721; dilution, 1:1,000) at room temperature for $2 \mathrm{~h}$. Next, the membranes were exposed using a Millipore enhanced chemiluminescence kit.

MTT assay. The proliferation rates of different U87 and U251 cells (Blank, vector and CADM2 groups) were detected using an MTT assay. A total of $1 \times 10^{4}$ cells/well were suspended in $200 \mu \mathrm{l}$ culture DMEM supplemented with $10 \%$ FBS and seeded in 96-well plates. Every $24 \mathrm{~h}$ after cell seeding, the culture medium of the 96-well plate was replaced with DMEM, and then incubated at $37^{\circ} \mathrm{C}$ for $4 \mathrm{~h}$. Next, DMEM was replaced with isopyknic dimethyl sulfoxide. Following 5 shocks, each of $1 \mathrm{sec}$ duration, the absorbance at $490 \mathrm{~nm}$ was detected by SpectraMax 190 (Molecular Devices, LLC, Sunnyvale, CA, USA) to measure the optical density (OD) value.

Wound-healing assay. Wound-healing assay was performed in 6 -well culture plates. In brief, $5 \times 10^{5}$ cells were suspended in medium containing $10 \% \mathrm{FBS}$, and were then seeded into 6-well plates until monolayer cells were nearly confluent. A $200-\mu 1$ sterile pipette tip was used to equably wound the monolayer cells, and the cells were washed with PBS twice to remove the floating cells. Following incubation in DMEM supplemented with $1 \%$ FBS for $24 \mathrm{~h}$, images of the wounded monolayers of blank, vector and CADM2 glioma cell groups were captured under a phase-contrast microscope (magnification, x20; Olympus Corporation, Tokyo, Japan).

Transwell invasion assay. QCM 24-Well Cell Invasion assay kit (EMD Millipore, Billerica, MA, USA) was used for the Transwell invasion assay processed by a Transwell filter coated 
A

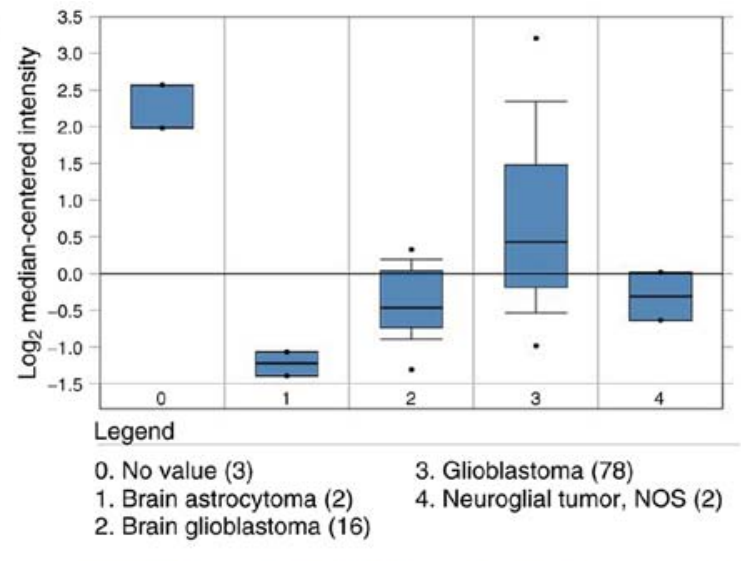

B

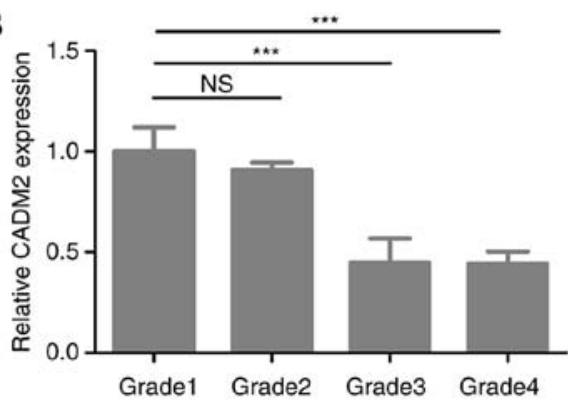

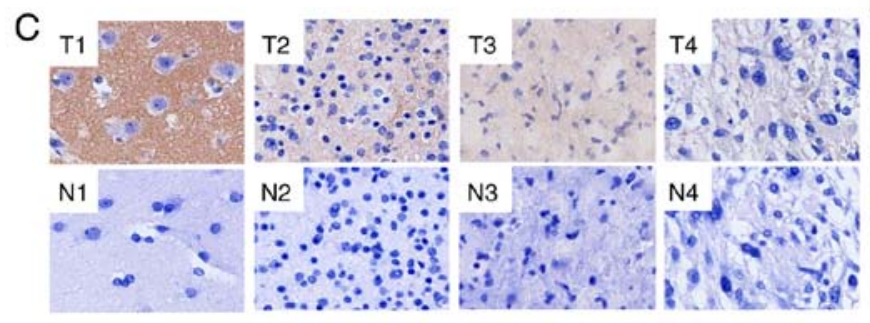

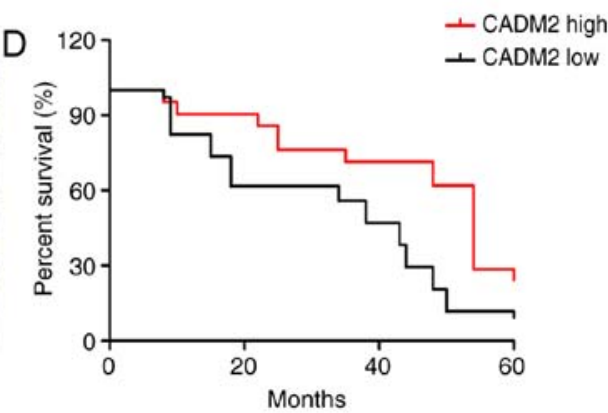

Figure 1. Bioinformatics analysis of CADM2 expression in human glioma. (A) CADM2 expression in the Oncomine database. (B) Tissue microarray analysis of CADM2 expression in human tissues. (C) Representative images of tissue microarray glioma tissue immunohistochemical staining (T1, T2, T3 and T4 represent no. 79, no. 20 , no. 23 and no. 41 of the microarray tissues, which are normal brain, and grade II, grade III and grade IV glioma tissues, respectively. N1, N2, N3 and N4 represent the corresponding adjacent normal tissues). (D) Kaplan-Meier survival curves of patients with glioma based on the protein expression levels of CADM2. ${ }^{*} \mathrm{P}<0.05$ and ${ }^{* * *} \mathrm{P}<0.001$. CADM2, cell adhesion molecule 2; ns, not significant; $\mathrm{T}$, tumor; $\mathrm{N}$, non-tumor.

with Matrigel. Cells were seeded onto the top side of the Transwell filter on the top chamber. DMEM supplemented with $10 \%$ FBS was added to the bottom chamber. Cells $\left(0.5 \times 10^{5}\right)$ were suspended in $250 \mu 1$ serum-free DMEM. After $72 \mathrm{~h}$ incubation, the residual cells were removed from the top chamber with a cotton swab, and the invaded cells were dislodged from the lower membrane surface using detachment solution. The detachment cells were stained with Hoechst 33342 at $37^{\circ} \mathrm{C}$ for $30 \mathrm{~min}$, and then images of the detached cells were directly captured and the number of cells was counted under a fluorescence microscope (magnification, $\mathrm{x} 20$ ). Cells were counted in at least 5 randomly selected fields of view for each group. The number of invaded cells per field was calculated and data are presented as the mean \pm standard error of the mean.

Animal studies. The stably transfected negative control (NC) and CADM2 lentivirus U87 cells $\left(2 \times 10^{6}\right.$ of each) were suspended in PBS and then implanted subcutaneously into 10 male Balb/c nude mice aged 4 weeks. The mice were purchased from Vital River Laboratories Co., Ltd. (Beijing, China). The length (a) and width (b) of NC and CADM2 mice tumors were monitored every 2 days. After 3 days of experiments, the nude mice were sacrificed and the weights of the animals ranged between 24.3 and $25.8 \mathrm{~g}$. Tumor volume (V) was calculated using the formula $\mathrm{V}=\mathrm{ab}^{2} / 2$. Animal experiments in the present study had been approved by the Ethics Committee of Tangdu Hospital Institutional Review Board.

Statistical analysis. Data are presented as the mean \pm standard deviation of at least three independent experiments. Significance between groups was analyzed by one-way analysis of variance, followed by a Newman-Keuls comparison test using GraphPad Prism version 5.03 (GraphPad Software, Inc., La Jolla, CA, USA). ${ }^{*} \mathrm{P}<0.05,{ }^{* *} \mathrm{P}<0.01$ and ${ }^{* * *} \mathrm{P}<0.001$ were considered to indicate statistically significant differences.

\section{Results}

CADM2 expression is downregulated in human glioma and predicts a poor prognosis in patients with glioma. To investigate the candidate role of CADM2 in human glioma, the data of CADM2 in the Oncomine database was initially referred to. It was demonstrated that the mRNA expression level of CADM2 was markedly decreased in tumor tissues (98 samples) compared with normal brain tissues (3 samples; Fig. 1A). Tissue microarrays were performed on 60 glioma tissues (grade I, II, III and IV), 10 cancer adjacent non-cancerous brain tissues and 10 normal brain tissues to compare the protein expression level of CADM2 in normal tissues and different grades of the malignancy (Fig. 1A). Tissue microarray results demonstrated that CADM2 expression was much lower in higher grade tissues, particularly grade II, III and IV glioma samples, than in the grade I samples (Fig. 1B). The CADM2 expression levels in different grades of glioma tissues and normal tissues were studied by immunohistochemistry. Representative images of these tissues are presented in Fig. 1C. Next, survival analysis was performed to study the association between CADM2 expression and survival times in patients with glioma. The Kaplan-Meier survival curves presented in Fig. 1D revealed that glioma patients with high CADM2 expression had a longer overall survival time than those with low CADM2 
A

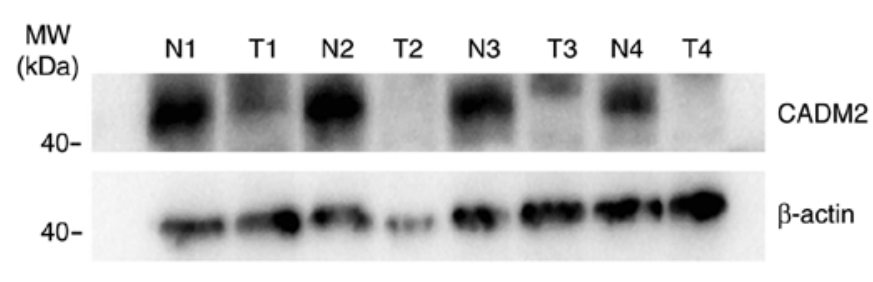

B

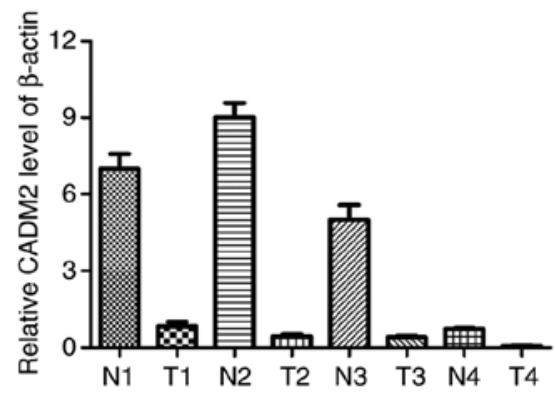

C

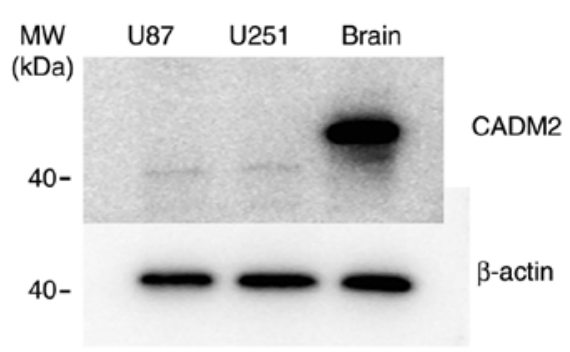

D

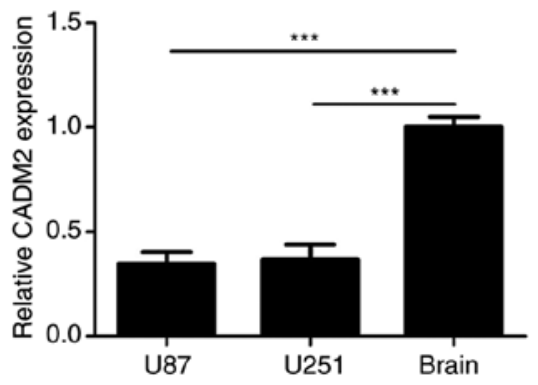

Figure 2. CADM2 is downregulated in human glioma tissues and cells. (A) Western blot analysis detected CADM2 expression in glioma tissues and adjacent normal tissues. (B) Quantitative analysis of CADM2 expression in glioma brain and GBM cell lines. (C) Western blot analysis detected CADM2 expression in glioma brain and GBM cell lines. (D) Reverse transcription-quantitative polymerase chain reaction detected CADM2 expression in glioma brain and GBM cell lines. ${ }^{* * *} \mathrm{P}<0.001$, unpaired two-tailed Student's t-test. CADM2, cell adhesion molecule 2; GBM, glioblastoma; MW, molecular weight.

expression $(\mathrm{P}<0.05)$. Western blot analysis also verified the lower expression level of CADM2 in 4 GBM tissues, compared with the 4 corresponding adjacent non-cancerous brain tissues (Fig. 2A and B). The results also demonstrated that CADM2 exhibited a much lower expression in glioma tumor tissues. In addition, CADM2 protein expression in the U87 and U251 cells was almost undetectable, while in normal brain cells it was particularly high (Fig. 2C), and the mRNA expression level of CADM2 in the GBM U87 and U251 cell lines was $\sim 60 \%$ lower than in the normal brain (Fig. 2D). Taken together, these data indicated that the low expression level of CADM2 at the mRNA and protein expression levels was observed in human glioma.

Downregulation of CADM2 is associated with aggressive tumor progression of human gliomas. To verify the association between CADM2 expression and GBM development, all 98 patients were stratified into CADM2-low and CADM2-high groups using the median value of CADM2 expression. Of the 98 patients with gliomas, $80(81.63 \%)$ belonged to the CADM2-low and $18(18.37 \%)$ to the CADM2-high groups, respectively. As demonstrated in Table I, low CADM2 expression occurred more frequently in glioma patients with advanced WHO grade (II-IV). However, no statistically significant differences were observed between CADM2 expression and other demographic characteristics, including patient age and sex $(\mathrm{P}>0.05$; Table I).

CADM2 inhibits glioma cell proliferation in vitro and in vivo. To investigate the biological function of CADM2 on glioma tumorigenesis, CADM2 was overexpressed in U87 and U251 cell lines, respectively (Fig. 3A and C). The effect of CADM2 on glioma cell proliferation was measured using an MTT cell proliferation assay. The results demonstrated that the overexpression of CADM2 significantly inhibited the proliferation of U87 and U251 compared with the blank group and the vector group (Fig. 3B and D). Nude mice xenograft tumors were created to verify the effect of CADM2 on glioma proliferation in vivo. The CADM2-overexpressing U87MG cells and the control U87MG cells were suspended in PBS and implanted into two sides of the mice. After 30 days, the tumor volume results demonstrated that CADM2 significantly inhibited U87MG xenograft tumor growth in vivo. The mean volumes of CADM2-U87 tumors cells were almost half the size of that of the control U87MG cells ( $\mathrm{n}=10$ animals per group, Fig. 3E and F). $\mathrm{Ki}-67$ staining revealed that there were fewer Ki-67-positive cells in CADM2-U87MG tumors than in U87MG control tumors (Fig.3Gand $\mathrm{H}$ ). Therefore, these results demonstrated that the CADM2 overexpression significantly inhibited glioma proliferation in vitro and in vivo.

CADM2 overexpression inhibits glioma cell migration and invasion. Next, a wound-healing assay and a Transwell assay were performed to study the role of CADM2 on glioma cell migration and invasion. In the wound-healing assay, the healing rate was calculated by the healing distance divided by the initial distance in order to demonstrate the cell migration ability. The results indicated that CADM2 inhibited the healing rate of glioma cells, and the inhibition rate of U87 and U251 cells was $57.6 \%$ (Fig. 4A and B) and $52.5 \%$ (Fig. 4C and D), respectively. The Transwell experiment was processed to examine the migratory capacity of glioma cells, and at least three wells were used for each experiment. The results demonstrated that the overexpression of CADM2 markedly attenuated the invasive properties of U87 and U251 cells; the number of invaded cells per field declined from 62 to 38/field for U87 cells (Fig. 4E and G) and from 52 to 25/field for U251 cells (Fig. 4F and H), respectively. The 
Table I. Associations between CADM2 protein expression and various clinicopathological characteristics of patients with glioma.

\begin{tabular}{|c|c|c|c|c|}
\hline Clinicopathological feature & No. cases & Low CADM2 expression, n (\%) & High CADM2 expression, n (\%) & P-value \\
\hline WHO grade & & & & $<0.001$ \\
\hline IV & 38 & $36(94.74)$ & $2(5.26)$ & \\
\hline III & 29 & $26(89.66)$ & $3(10.34)$ & \\
\hline II & 17 & $14(82.35)$ & $3(17.65)$ & \\
\hline I & 14 & $4(28.57)$ & $10(71.43)$ & \\
\hline Age, years & & & & 0.352 \\
\hline$<55$ & 44 & $39(88.64)$ & $5(11.36)$ & \\
\hline$\geq 55$ & 54 & $47(87.04)$ & $7(12.96)$ & \\
\hline Sex & & & & 0.438 \\
\hline Male & 55 & $49(89.09)$ & $6(10.91)$ & \\
\hline Female & 43 & $38(88.37)$ & $5(11.63)$ & \\
\hline KPS score & & & & 0.025 \\
\hline$<80$ & 45 & $38(84.44)$ & $7(5.56)$ & \\
\hline$\geq 80$ & 53 & $45(84.91)$ & $8(5.09)$ & \\
\hline
\end{tabular}

WHO, World Health Organization; KPS, Karnofsky Performance Status.

A

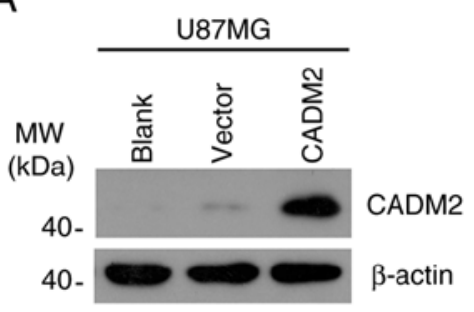

B

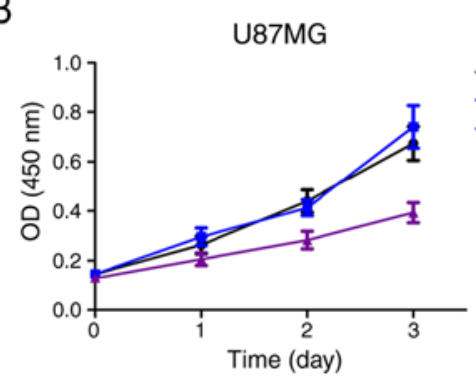

C

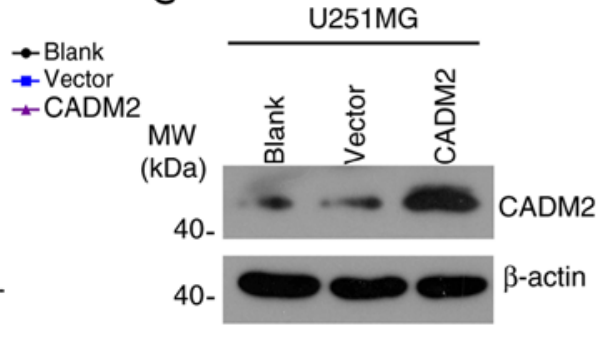

D

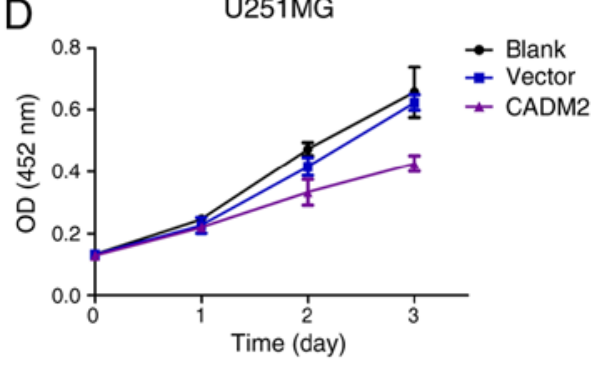

G

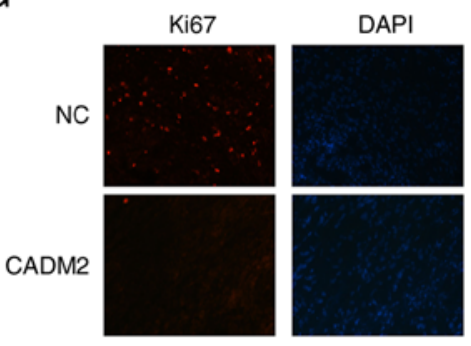

E

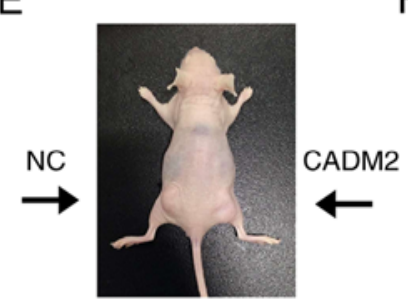

F

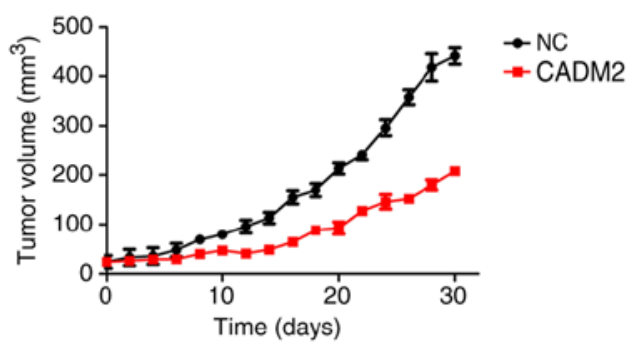

$\mathrm{H}$

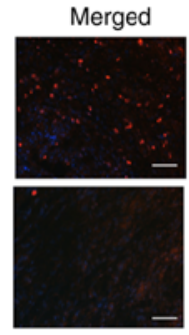

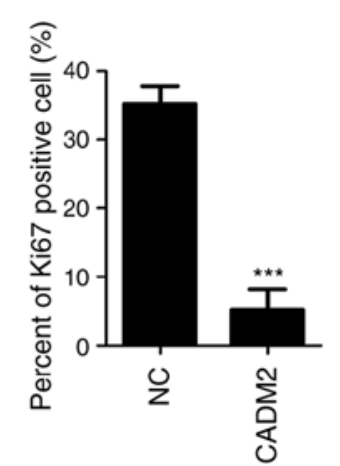

Figure 3. CADM2 inhibits glioma cell proliferation in vitro and in vivo. (A) Western blot analysis detected CADM2-overexpression in U87 cells. (B) Growth curve of U87 cells by MTT assay. (C) Western blot analysis detected CADM2-overexpression in U251 cells. (D) Growth curve of U251 cells by MTT assay. (E) Representative image of xenograft mice. Scale bar, $200 \mu \mathrm{m}$. (F) The growth curve of tumorigenesis. The tumor volume was recorded every 2 days. (G) Representative images of Ki-67 (red) expression levels in xenografted tumors, DAPI was used for nuclei staining. (H) Quantitative analysis of the Ki-67 positive cells. ${ }^{* * *} \mathrm{P}<0.001$. CADM2, cell adhesion molecule 2 ; OD, optical density. 
A
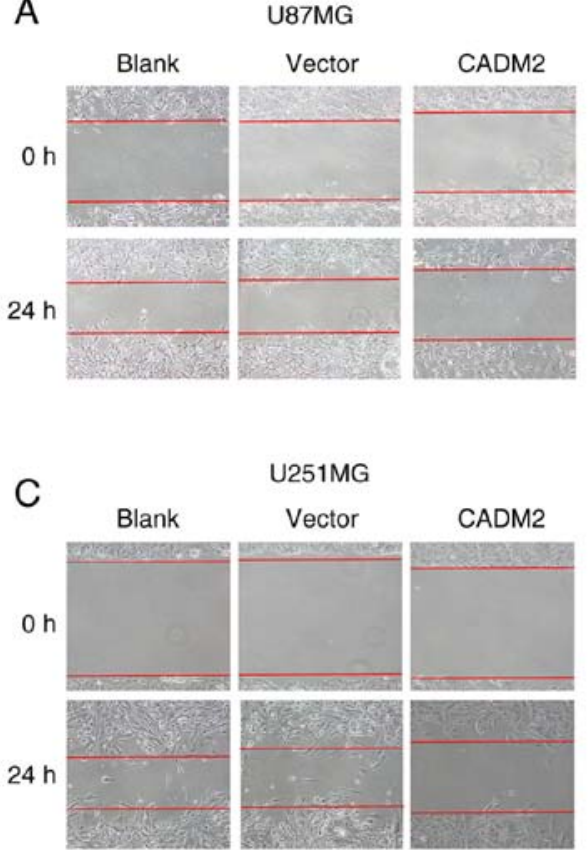

U251MG

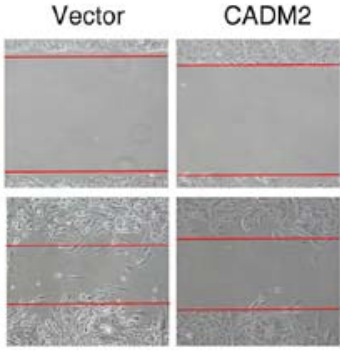

B

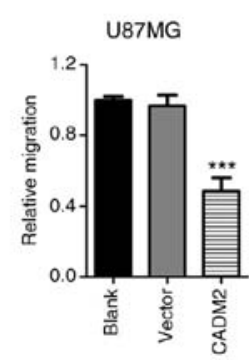

D

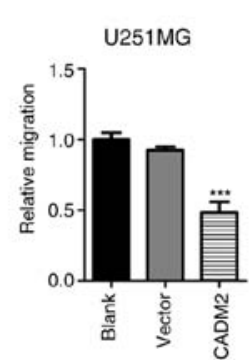

E
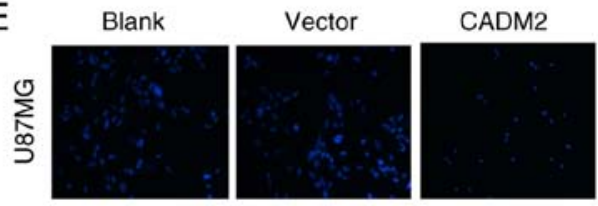

F

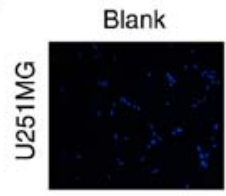

Vector

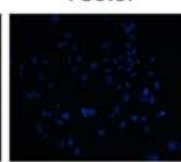

CADM2

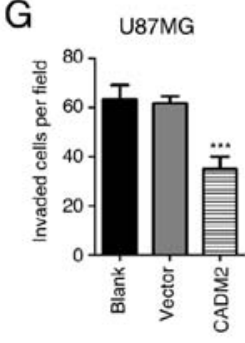

$\mathrm{H}$

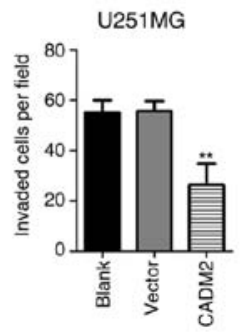

Figure 4. CADM2 inhibits glioma cell migration and invasion. (A) Representative images of wound healing assay of U87 cells. (B) Quantitative analysis of U87 wound healing rate. (C) Representative images of wound healing assay of U251 cells. (D) Quantitative analysis of U251 wound healing rate. (E) Representative images of Tranwell assay of U87 cells. (F) Representative images of Transwell assay of U251 cells. (G) Quantitative analysis of invaded U87 cells. (H) Quantitative analysis of invaded U251 cells. DAPI was used for invaded cell staining. Error bars represent the standard error of the mean. ${ }^{* *} \mathrm{P}<0.01,{ }^{* * *} \mathrm{P}<0.001$. CADM2, cell adhesion molecule 2 .
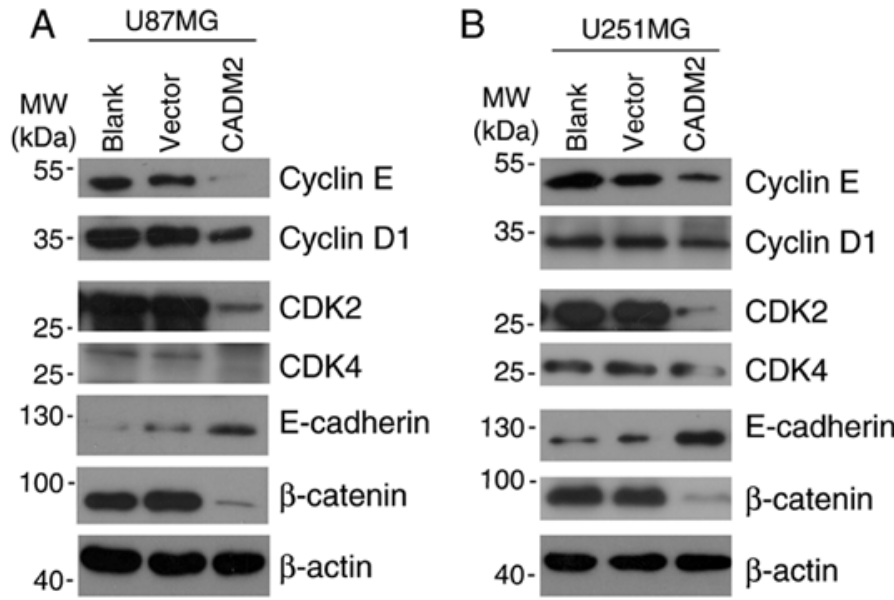

Figure 5. CADM2 regulates the expression of cell cycle-related genes and EMT-related genes. (A) Cell cycle-related genes and EMT-related genes in U87 cells detected by western blot analysis. (B) Cell cycle-related genes and EMT-related genes in U251 cells detected by western blot analysis. CADM2, cell adhesion molecule 2; CDK, cyclin-dependent kinase; EMT, epithelial-mesenchymal transition.

results indicated that CADM2 could inhibit the migratory and invasive abilities of glioma cells.

CADM2 regulates the cell cycle and EMT-related genes in glioma cells. CADMs were reported as key regulators in G1/S transition. The results of the present study demonstrated that CADM2 overexpression significantly decreased the protein expression levels of cyclin D1, CDK4, cyclin E1 and CDK2, compared with the blank group (Fig. 5A and B). The EMT is a crucial process for the invasion of cancer cells $(17,18)$. Next, the expression of two EMT markers, E-cadherin and $\beta$-catenin, was detected by western blot analysis. The results demonstrated that
CADM2 significantly downregulated the protein expression level of $\beta$-catenin and markedly increased the expression level of E-cadherin in U87 cells (Fig. 5A) and U251 cells (Fig. 5B). These data suggested that CADM2 may inhibit glioma cell proliferation by regulating cell cycle related genes and inhibit glioma cell invasion via regulating the glioma cell EMT process.

\section{Discussion}

Glioma is one of the most common primary malignant tumors of the brain parenchyma, which is also one of the most difficult types of cancer to cure. Although a large number of 
advances have been achieved in surgery, radiation therapy and chemotherapy over recent decades, the prognosis of malignant glioma remains poor. Comprehensive treatment with specific molecular targets is thought to be a promising therapeutic strategy. Therefore, it is urgent to identify novel and efficient targets for glioma therapy.

Recent studies have reported that the CADMs family function as tumor suppressors $(6,7)$. In numerous types of malignant tumors, the CADMs family are not expressed or are only expressed at a low level. For example, CADM1 is reported to be downregulated in lung (19), prostate (20), esophageal (21) and breast cancer (22). CADM3 and CADM4 have also been reported to suppress the growth of numerous types of cancer (23-25). Among the CADMs family, the role of CADM2, as well as that of other members, in cancer has not yet been characterized (10). According to the limited number of previous studies, CADM2 may participate in maintaining cell polarity and decrease the adhesion of renal cell carcinoma (cRCC) (6). The downregulation of CADM2 in $\mathrm{cRCC}$ is probably due to promoter methylation, and the CADM2 promoter methylation is associated with tumor grade in primary cRCC (6). Promoter methylation may mediate the mechanism by which CADM2 downregulation occurs in cRCC. A previous study demonstrated that CADM2 promoter methylation was correlated with tumor stage and grade in primary $\mathrm{cRCC}$, and the expression of CADM2 was restored in selected cell lines following 5-azadC treatment (6). However, there is no clear conclusion regarding the expression and clinical significance of CADM2 in human brain gliomas so far.

To the best of our knowledge, the present study was the first regarding the biological function of CADM2 in human glioma. Based on tissue microarray analysis, combined with qPCR detection in glioma tissues and cell lines, it was demonstrated that CADM2 is significantly downregulated in human glioma. The expression intensity of CADM2 was significantly correlated with patient pathological grade. The present study demonstrated that CADM2 overexpression inhibited glioma cell proliferation in vitro and in vivo. In addition, CADM2 also markedly inhibited the migration and invasion of glioma cells. It was further demonstrated that CADM2 regulated the expression of cell cycle associated genes and EMT associated genes. In conclusion, CADM 2 inhibits glioma cell proliferation, migration and invasion by regulating the glioma cell cycle and EMT. CADM2 may be a novel therapeutic target for glioma treatment.

\section{Acknowledgements}

Not applicable.

\section{Funding}

The present study was supported by the National Natural Scientific Foundation of China (grant no. 81572983), the Foundation of Science Innovation and Development in Tangdu Hospital, Fourth Military Medical University (grant no. 2016JCYJ013), the Natural Scientific Foundation of Shaanxi Province (grant no. 2014JM4148) and the Beijing Key Laboratory of Brain Major Diseases Open Project (grant no. 2015NZDJ02).

\section{Availability of data and materials}

All data generated and/or analyzed during this study are included in this published article.

\section{Authors' contributions}

YT and YC conceived and designed the study; NL performed the majority of the experiments and wrote the majority of the manuscript; CY and WB collected clinical information, performed statistical analyses and wrote part of the manuscript; $\mathrm{XW}$ and MJ also gave academic guidance during the process of experimental design; HY gave guidance and constructive suggestions in the animal experiment section; $\mathrm{ZW}, \mathrm{WW}$, PZ and HL assisted with PCR, western blotting and in vitro experiments. All authors read and approved the manuscript, and agree to be accountable for all aspects of the research in ensuring that the accuracy or integrity of any part of the work are appropriately investigated and resolved.

\section{Ethics approval and consent to participate}

The present study was approved by the Ethics Committee of Tangdu Hospital, and written informed consent was obtained from all patients prior to sample collection.

\section{Patient consent for publication}

Not applicable.

\section{Competing interests}

The authors state that they have no competing interests.

\section{References}

1. Dunn GP, Rinne ML, Wykosky J, Genovese G, Quayle SN, Dunn IF, Agarwalla PK, Chheda MG, Campos B, Wang A, et al: Emerging insights into the molecular and cellular basis of glioblastoma. Genes Dev 26: 756-784, 2012.

2. Louis DN, Ohgaki H, Wiestler OD, Cavenee WK, Burger PC, Jouvet A, Scheithauer BW and Kleihues P: The 2007 WHO classification of tumours of the central nervous system. Acta Neuropathol 114: 97-109, 2007.

3. Oike T, Suzuki Y, Sugawara K, Shirai K, Noda SE, Tamaki T, Nagaishi M, Yokoo H, Nakazato Y and Nakano T: Radiotherapy plus concomitant adjuvant temozolomide for glioblastoma: Japanese mono-institutional results. PLoS One 8: e78943, 2013.

4. Fiorica F, Carau B, Ursino S and Cartei F: Radiotherapy plus concomitant and adjuvant temozolomide for unresectable glioblastoma: A retrospective analysis of our experience. Radiother Oncol 81: S297-S297, 2006

5. Hargreaves SJ, Williams M, Liu Z, et al: Survival in patients receiving radiotherapy plus concomitant and adjuvant temozolomide (RCAT) for glioblastoma (GBM). Int J Radiat Oncol Biol Phys 78: S281-S282, 2010.

6. He W, Li X, Xu S, Ai J, Gong Y, Gregg JL, Guan R, Qiu W, Xin D, Gingrich JR, et al: Aberrant methylation and loss of $C A D M 2$ tumor suppressor expression is associated with human renal cell carcinoma tumor progression. Biochem Biophys Res Commun 435: 526-532, 2013.

7. Yang S, Yan HL, Tao QF, Yuan SX, Tang GN, Yang Y, Wang LL, Zhang YL, Sun SH and Zhou WP: Low CADM2 expression predicts high recurrence risk of hepatocellular carcinoma patients after hepatectomy. J Cancer Res Clin Oncol 140: 109-116, 2014.

8. Takai Y, Irie K, Shimizu K, Sakisaka T and Ikeda W: Nectins and nectin-like molecules: Roles in cell adhesion, migration, and polarization. Cancer Sci 94: 655-667, 2003. 
9. Murakami Y: Involvement of a cell adhesion molecule, TSLC1/IGSF4, in human oncogenesis. Cancer Sci 96: 543-552, 2005.

10. Chang GM, Xu SP, Dhir R, Chandran U, O'Keefe DS, Greenberg NM and Gingrich JR: Hypoexpression and epigenetic regulation of candidate tumor suppressor gene $C A D M-2$ in human prostate cancer. Clin Cancer Res 16: 5390-5401, 2010.

11. Cody NA, Shen Z, Ripeau JS, Provencher DM, Mes-Masson AM, Chevrette $\mathrm{M}$ and Tonin PN: Characterization of the 3p12.3-pcen region associated with tumor suppression in a novel ovarian Cancer cell line model genetically modified by chromosome 3 fragment transfer. Mol Carcinog 48: 1077-1092, 2009.

12. Lake SL, Coupland SE, Taktak AF and Damato BE: Whole-genome microarray detects deletions and loss of heterozygosity of chromosome 3 occurring exclusively in metastasizing uveal melanoma. Invest Ophthalmol Vis Sci 51: 4884-4891, 2010.

13. Roy D, Sin SH, Damania B and Dittmer DP: Tumor suppressor genes FHIT and WWOX are deleted in primary effusion lymphoma (PEL) cell lines. Blood 118: e32-e39, 2011

14. Tu YY, Gao XC, Li G, Fu H, Cui D, Liu H, Jin W and Zhang Y: MicroRNA-218 inhibits glioma invasion, migration, proliferation, and cancer stem-like cell self-renewal by targeting the polycomb group gene Bmil. Cancer Res 73: 6046-6055, 2013

15. Allen M, Bjerke M, Edlund $H$, Nelander $S$ and Westermark B: Origin of the U87MG glioma cell line: Good news and bad news. Sci Transl Med 8: 354re3, 2016.

16. Livak KJ and Schmittgen TD: Analysis of relative gene expression data using real-time quantitative PCR and the $2^{-\Delta \Delta C \mathrm{~T}}$ method. Methods 25: 402-408, 2001.

17. Micalizzi DS, Farabaugh SM and Ford HL: Epithelialmesenchymal transition in cancer: Parallels between normal development and tumor progression. J Mammary Gland Biol Neoplasia 15: 117-134, 2010.
18. Lee MY, Chou CY, Tang MJ and Shen MR: Epithelial mesenchymal transition in cervical cancer: Correlation with tumor progression, epidermal growth factor receptor overexpression, and snail up-regulation. Clin Cancer Res 14: 47-43, 2008.

19. Kuramochi M, Fukuhara H, Nobukuni T, Kanbe T, Maruyama T, Ghosh HP, Pletcher M, Isomura M, Onizuka M, Kitamura T, et al: TSLC1 is a tumor-suppressor gene in human non-small-cell lung cancer. Nat Genet 27: 427-430, 2001

20. Fukuhara H, Kuramochi M, Fukami T, Kasahara K, Furuhata M, Nobukuni T, Maruyama T, Isogai K, Sekiya T, Shuin T, et al: Promoter methylation of TSLCl and tumor suppression by its gene product in human prostate cancer. Jap J Cancer Res 93: 605-609, 2002

21. Ito T, Shimada Y, Hashimoto Y, Kaganoi J, Kan T, Watanabe G, Murakami Y and Imamura M: Involvement of TSLC1 in progression of esophageal squamous cell carcinoma. Cancer Res 63: 6320-6326, 2003.

22. Takahashi Y, Iwai M, Kawai T, Arakawa A, Ito T, SakuraiYageta M, Ito A, Goto A, Saito M, Kasumi F, et al: Aberrant expression of tumor suppressors CADM1 and 4.1B in invasive lesions of primary breast cancer. Breast Cancer 19: 242-252, 2012.

23. Williams YN, Masuda M, Sakurai-Yageta M, Maruyama T, Shibuya $M$ and Murakami Y: Cell adhesion and prostate tumor-suppressor activity of TSLL2/IGSF4C, an immunoglobulin superfamily molecule homologous to TSLC1/IGSF4. Oncogene 25: 1446-1453, 2006.

24. Raveh S, Gavert N, Spiegel I and Ben-Ze'ev A: The cell adhesion nectin-like molecules (Necl) 1 and 4 suppress the growth and tumorigenic ability of colon cancer cells. J Cell Biochem 108: 326-36, 2009.

25. Nagata M, Sakurai-Yageta M, Yamada D, Goto A, Ito A, Fukuhara H, Kume H, Morikawa T, Fukayama M, Homma Y, et al: Aberrations of a cell adhesion molecule CADM4 in renal clear cell carcinoma. Int J Cancer 130: 1329-1337, 2012. 